\title{
How Does Smoking and Nicotine Dependence Change After Onset of Vaping? A Retrospective Analysis of Dual Users
}

\author{
Úrsula Martínez PhD ${ }^{1,0}$, Víctor Martínez-Loredo PhD², \\ Vani N. Simmons PhD ${ }^{1,3,4}$, Lauren R. Meltzer MPH ${ }^{1,0}$, \\ David J. Drobes PhD ${ }^{1,3,4}$, Karen O. Brandon PhD', Amanda M. Palmer \\ $\mathrm{MA}^{1,3}$, Thomas Eissenberg PhD ${ }^{5}$, Christopher R. Bullen PhD ${ }^{6, \ominus}$, \\ Paul T. Harrell PhD7, Thomas H. Brandon, PhD ${ }^{1,3,4}$
}

\begin{abstract}
'Department of Health Outcomes and Behavior, H. Lee Moffitt Cancer Center, Tampa, FL; ${ }^{2}$ Clinical Unit of Addictive Behaviors, Department of Psychology, University of Oviedo, Spain; ${ }^{3}$ Department of Psychology, University of South Florida, Tampa, FL; ${ }^{4}$ Department of Oncologic Sciences, University of South Florida, Tampa, FL; ${ }^{5}$ Center for the Study of Tobacco Products, Department of Psychology, Virginia Commonwealth University, Richmond, VA; ${ }^{6}$ School of Population Health, Faculty of Medical and Health Science, University of Auckland, New Zealand; ' ${ }^{2}$ epartment of Pediatrics, Eastern Virginia Medical School, Norfolk, VA
\end{abstract}

Corresponding Author: Úrsula Martínez, PhD, Tobacco Research and Intervention Program, Moffitt Cancer Center, $4115 \mathrm{E}$ Fowler Avenue, Tampa, FL 33617, USA. Telephone: 813-745-1754; E-mail: Ursula.martinez@moffitt.org

\begin{abstract}
Introduction: Although some smokers switch to exclusive use of electronic cigarettes (e-cigarettes), others become dual users of combustible cigarettes and e-cigarettes. Little is known about how the onset of vaping affects the use of and dependence on combustible cigarettes or total nicotine use and dependence, which may influence health-related and cessation outcomes. Using self-report data of current combustible and e-cigarette use and retrospective recall of pre-vaping smoking in a sample of dual users, the aims of this study were (1) to compare pre- and post-vaping number of cigarettes per day and combustible cigarette dependence; (2) to compare pre- and post-vaping total nicotine use frequency (number of vaping sessions and cigarettes smoked per day), and total nicotine dependence; and (3) to examine predictors of nicotine dependence.

Methods: We used baseline data from a smoking cessation trial with 2896 dual users. Nicotine use frequency and the Heaviness of Smoking Index were used as measures of nicotine use and dependence, respectively.

Results: Participants decreased cigarettes/day from pre- $(M=19.24, S D=9.01)$ to post-vaping $(M=11.15, S D=8.02, p<.0001)$ and combustible cigarette dependence declined from pre- $(M=3.55$, $S D=1.51)$ to post-vaping $(M=2.11, S D=1.60, p<.0001)$. Total daily nicotine use frequency increased after initiating vaping $(M=19.25, S D=9.01$ vs. $M=29.46, S D=8.61 ; p<.0001)$, as did total nicotine dependence $(M=3.55, S D=1.51$ vs. $M=4.68, S D=1.38 ; p<.0001)$. Hierarchical regression analyses indicated that variables associated with greater overall nicotine dependence included: younger age, lower education, more years smoking, higher pre-vaping nicotine dependence, using e-cigarettes more days per month, more puffs per vaping session, higher e-liquid nicotine concentration, and longer vaping history.
\end{abstract}




\section{Conclusions: Dual use leads to a reduction in the number of combustible cigarettes, but total nicotine use and dependence increases. \\ Implications: In dual users, a reduction in smoking following onset of vaping may offer some harm reduction via reduction in cigarette intake. However, the increase in total nicotine use and dependence could affect the ability to quit either or both products.}

\section{Introduction}

Electronic cigarettes (e-cigarettes) were developed in China in 2003 and have gained popularity worldwide over the past decade. Prevalence of e-cigarette use varies widely, with rates of $4 \%-6 \%$ in countries such as the United States or United Kingdom or $1 \%$ in Australia or Canada, which have more restrictive policies. ${ }^{1}$

The popularity of e-cigarettes may present an opportunity to decrease the health burden of tobacco smoking, given that they appear to be an appealing and potentially less lethal way of delivering nicotine than combustible cigarettes. ${ }^{2}$ For instance, smokers find that e-cigarettes have several attractive features relative to conventional nicotine replacement therapies (NRTs; eg, greater similarity to cigarette smoking, better reduction of cravings, less expensive). ${ }^{3,4}$ Despite these advantages, the efficacy of e-cigarettes for smoking cessation is still not clear, ${ }^{5}$ and concerns about e-cigarettes remain, including their potential as a gateway to tobacco use in youth, their unknown long-term health effects, and the possibility that they may impede or delay smoking cessation. ${ }^{6}$ The last concern is important because one of the primary reported reasons to start using e-cigarettes is to quit smoking. ${ }^{3,7}$ Population studies suggest that, given frequent daily use, e-cigarettes do increase smoking cessation rates. ${ }^{8-10}$ However, the only two randomized controlled trials published so far failed to find a significant effect of e-cigarettes on cessation, ${ }^{11,12}$ although a Cochrane meta-analysis of the studies found some support for cessation efficacy. ${ }^{13}$

The majority of e-cigarette users (vapers) are "dual users"-that is, they continue to smoke combustible cigarettes. ${ }^{14}$ Population-based surveys of adults have consistently found that the highest prevalence of e-cigarette use is among current smokers, ${ }^{15,16}$ with many $(72 \%-$ $89 \%)^{17,18}$ persisting in this dual-use pattern a year later, although they are likely to report an intention to quit smoking. ${ }^{19,20}$ The association between dual use and smoking cessation has produced mixed findings. ${ }^{18,20,21}$ For example, some studies found that dual use was associated with greater smoking cessation over time compared to smokers not using e-cigarettes. ${ }^{22,23}$ However, prospective cohort studies of smokers have not found e-cigarette use to be associated with greater rates of smoking cessation. ${ }^{21,24}$

One of the key concerns regarding dual use of tobacco cigarettes and e-cigarettes is nicotine dependence. Because dual users receive nicotine from both products, they may become more nicotine dependent, ${ }^{25}$ and thus delay achieving smoking abstinence. ${ }^{26,27}$ Very few studies have investigated nicotine dependence in dual users, and in those that have, most have been cross-sectional and focused on combustible cigarette dependence. For example, using a modified version of the Fagerström Test for Nicotine Dependence in a sample of 319 participants $(33 \%$ exclusive vapers, $37 \%$ smokers, and $30 \%$ dual users) Ruther et al. ${ }^{27}$ found that dual users had higher nicotine dependence than exclusive smokers or exclusive vapers. ${ }^{27}$ Rostron et al. ${ }^{25}$ compared exclusive daily combustible cigarette $(n=3963)$ with dual $(n=774)$ users and found no significant differences in the number of cigarettes smoked per day and time to first cigarette smoked. However, they excluded non-daily cigarette smokers in their sample. Interestingly, dual users were more likely to report greater levels of craving and withdrawal symptoms. Nardone et al. ${ }^{28}$ compared nicotine dependence among exclusive e-cigarette users $(n=68)$, light dual users (smoking $\leq 5$ combustible cigarettes per day; $n=31$ ), and heavy dual users (smoking $>5$ combustible cigarettes per day; $n=69$ ). Overall, they found that nicotine intake was similar among groups. Finally, Morean et al. ${ }^{29}$ found that dual users $(n=326)$ were more dependent on combustible tobacco cigarettes than e-cigarettes.

Overall, these prior studies suggest that dual users may be more nicotine dependent than exclusive smokers or exclusive vapers. However, the direction of causality is not clear from the previously described cross-sectional studies. That is, it is unknown whether the onset of vaping in smokers (ie, dual use) led to higher nicotine dependence or if higher nicotine dependence before vaping led to the use of e-cigarettes. Thus, more information is needed about the transition from cigarette smoking to vaping onset and dual use.

In this study, we used current and retrospective self-reports of smoking and vaping patterns in a sample of dual users to (1) compare pre- and post-vaping smoking rates (ie, cigarettes per day) and smoking-related nicotine dependence; (2) compare pre- and post-vaping total nicotine frequency (number of vaping sessions and cigarettes smoked per day), and combined nicotine dependence; and (3) examine predictors of tobacco cigarette dependence and combined nicotine dependence after initiating vaping.

Although previous studies have reported combustible or e-cigarette nicotine dependence before and after the onset of vaping, ${ }^{30-33}$ they have not attended to the combined nicotine usage or dependence associated with both products. Thus, to our knowledge, this is the first study that combines combustible cigarettes and e-cigarettes in a single measure to compare pre- and post-vaping nicotine dependence.

\section{Methods}

This study is a secondary analysis of baseline data from a smoking cessation trial investigating the efficacy of a self-help intervention designed to promote smoking cessation and eventual nicotine abstinence in dual users. ${ }^{34}$

\section{Participants}

Participants were 2896 dual users who were not necessarily seeking smoking cessation treatment and who (1) smoked at least 1 cigarette per week over the past year; (2) used an e-cigarette at least 1 time per week over the past month; (3) were at least 18 years of age; (4) were able to speak and write English; and (5) were not currently enrolled in a face-to-face smoking cessation treatment. Participants receiving other smoking cessation treatments (eg, NRT) were not excluded from the study.

\section{Procedure}

Recruitment and study procedures have been described in detail elsewhere. ${ }^{34}$ Participants were recruited throughout the United 
States between July 2016 and July 2017 using social media, online e-cigarette forums, and advertising via television and newspapers. Participants who contacted the study were screened for eligibility and asked for informed consent to participate in the study over the telephone. Those who were eligible and provided consent were sent a baseline questionnaire via mail or e-mail, according to their preference. Participants who returned the completed baseline questionnaires and still met inclusion criteria were formally enrolled in the study and compensated with $\$ 20$ for completing the assessment. The study protocol was approved by the Chesapeake Institutional Review Board.

\section{Measures}

Standard survey items assessed age, sex, education, marital status, income, race, and ethnicity. Participants' smoking and vaping history and behavior were also assessed, including the number of years of smoking before initiating vaping. Number of cigarettes smoked per day before and after e-cigarette use onset were reported using the following categories " 5 or less cigarettes per day," "6-10 cigarettes per day," "11-15 cigarettes per day," "16-20 cigarettes per day," "21-30 cigarettes per day,” and “+31 cigarettes per day.” For these analyses, responses were coded using the midpoint of each range, with the last category (+31 cigarettes per day) being coded as 35 .

Duration of e-cigarette use was categorized as "less than 1 month ago," "1-6 months ago," "7-12 months ago," "13-24 months ago," and "more than 24 months ago." Because of the small sample size in the two first categories, they were combined into one category for these analyses. Current frequency of use was assessed as "On days you use them, please estimate how many separate times per day you usually use your e-cigarette?" with the following response options: "1-4 times a day," "5-9 times a day," "10-14 times a day," "15-19 times a day," "20-29 times a day," "more than 30 times a day," and "I vape continuously throughout the day." Responses were coded using the midpoint of each range, with a value of 35 and 40 imputed, respectively for the last two categories.

Number of puffs per vaping session was assessed as "About how many e-cigarette puffs on average do you take each time you use an e-cigarette?" Response categories were "1-10 puffs," "11-20 puffs," "21-30 puffs," and "30+ puffs." Concentration or strength of nicotine in the e-liquid or cartridge of the e-cigarette was assessed using the categories "0 mg," "1-3 mg," “4-8 mg," "9-15 mg," "16-24 mg," "more than $24 \mathrm{mg}$," and "I don't know." Participants reporting "I don't know" were coded as missing for the regression analyses.

Nicotine use frequency and the Heaviness of Smoking Index $(\mathrm{HSI})^{35}$ were used as measures of nicotine dependence. For the assessment of nicotine frequency, we used number of cigarettes per day as the pre-vaping measure. For post-vaping nicotine frequency, we used the sum of current cigarettes per day and vaping sessions per day. Participants who reported using e-cigarettes with no nicotine were given a value of 0 for vaping sessions per day.

The HSI uses the two items of the Fagerström Test for Nicotine Dependence $^{36}$ that have shown to be most predictive of difficulty to quit smoking (ie, number of cigarettes per day, time to first cigarette). These two items have been also adapted to measure nicotine dependence in e-cigarette users in previous studies. ${ }^{20,33}$ In this study, the HSI was used for the assessment of nicotine dependence via combustible cigarette smoking pre- and post-vaping (HSI-cig). To assess total nicotine dependence (HSI-nic) post-vaping, we used time to first nicotine (either combustible or e-cigarette) and number of nicotine sessions per day (combustible cigarettes per day + vaping sessions per day). Participants who reported using e-cigarettes with no nicotine content were assigned a value of 0 vaping sessions per day and their time to first nicotine was based on their first combustible cigarette.

\section{Statistical Analyses}

Descriptive statistics were used to summarize participants' demographic information and smoking and vaping characteristics. Differences between measures of nicotine dependence pre- and post-vaping were analyzed using paired $t$-tests. Effect sizes were calculated using Cohen's $d$. Separate multiple hierarchical regression analyses were used to identify significant (Bonferroni adjusted $\alpha=$ .008) predictors of current combustible tobacco nicotine dependence (HSI-cig) and total nicotine dependence (HSI-nic). After checking for multicollinearity, variables were entered in the model as follows. In the first and second blocks, we included sociodemographic variables (sex, age, race, ethnicity, marital status, education, and income) and smoking characteristics (years smoking and pre-vaping HSI) associated with nicotine dependence, respectively. In the third block, we included e-cigarette variables that could be related to nicotine dependence and were on an interval scale (number of days per month vaping, number of puffs per vaping session, nicotine strength, and number of months using e-cigarettes). Data were analyzed using the IBM SPSS, version 24.0 statistical package.

\section{Results}

Of the 5821 participants assessed for eligibility, 3113 completed the baseline questionnaire and consented to participate in the study. Of those, 217 did not meet inclusion criteria at the time the baseline questionnaire was received, mostly because they did not smoke or vape enough. Thus, 2896 participants met inclusion criteria and were included in the study. As shown in Table 1, the final sample was mostly men, largely white, and the majority had a household income lower than US\$30 000. Participants had been smoking for more than a decade on average before using e-cigarettes. Before vaping, most participants reported smoking between 16 and 20 cigarettes per day and reported smoking the first cigarette of the day within the first 5 minutes. After vaping, more than half of participants smoked 10 cigarettes or less per day and most reported smoking their first cigarette of the day between 6 and 30 minutes after waking. Less than $1 \%$ of the sample was using NRT ( $n=17$ gum; $n=11$ patch; $n=1$ spray; and $n=9$ lozenges).

Table 2 summarizes e-cigarette use among the sample. Over half of the sample indicated that they started vaping more than 2 years earlier, $68.1 \%$ began vaping with the primary goal of quitting or reducing smoking, with another $12.1 \%$ who began vaping because of smoking health concerns. Participants most frequently reported using e-cigarettes daily, continuously throughout the day, taking between 1 and 10 puffs per session, and using relatively low nicotine concentrations $(0-3 \mathrm{mg})$. The most frequently used e-cigarette type was refillable tank systems.

Table 3 shows differences from before to after the onset of vaping in the use of and dependence on combustible cigarettes and total nicotine. All differences were significant at $p<.0001$. The number of cigarettes per day decreased by $42 \%$, with $79 \%$ of participants reporting a decline in smoking. Likewise, cigarette-based dependence (HSI-cig) decreased from pre- to post-vaping. However, the total nicotine use frequency and dependence (HSI-nic) increased from pre- to post-vaping. Overall, $71 \%$ of the sample reported increased 
Table 1. Demographic Variables and Smoking Characteristics

\begin{tabular}{|c|c|}
\hline & Mean $(\mathrm{SD})$ or $n(\%)$ \\
\hline \multicolumn{2}{|l|}{ Demographic variables } \\
\hline Sex (male) & $1830(63.2 \%)$ \\
\hline Age & $29.85(11.2)$ \\
\hline \multicolumn{2}{|l|}{ Race } \\
\hline White & $2550(93.4 \%)$ \\
\hline Black & $86(3.2 \%)$ \\
\hline Other & $94(3.4 \%)$ \\
\hline Ethnicity (Hispanic/Latino) & $241(8.3 \%)$ \\
\hline Marital status (married or has life partner) & $1001(34.6 \%)$ \\
\hline Education (beyond high school diploma) & $1524(52.6 \%)$ \\
\hline Household income (US\$ < 30000$)$ & $1636(56.7 \%)$ \\
\hline \multicolumn{2}{|l|}{ Smoking characteristics before e-cigarettes } \\
\hline Years smoking & $12.93(10.9)$ \\
\hline \multicolumn{2}{|l|}{ Cigarettes per day } \\
\hline 5 or less & $167(5.8 \%)$ \\
\hline $6-10$ & $432(14.9 \%)$ \\
\hline $11-15$ & $304(10.5 \%)$ \\
\hline $16-20$ & $907(31.3 \%)$ \\
\hline $21-30$ & $722(24.9 \%)$ \\
\hline$>30$ & $363(12.5 \%)$ \\
\hline \multicolumn{2}{|l|}{ First cigarette of the day } \\
\hline$\leq 5 \mathrm{~min}$ & $1368(47.3 \%)$ \\
\hline $6-30 \mathrm{~min}$ & $1088(37.6 \%)$ \\
\hline $31-60 \mathrm{~min}$ & $256(8.8 \%)$ \\
\hline$>60 \mathrm{~min}$ & $182(6.3 \%)$ \\
\hline Heaviness of Smoking Index (HSI-cig) & $3.55(1.5 \%)$ \\
\hline \multicolumn{2}{|l|}{ Smoking characteristics after e-cigarettes } \\
\hline \multicolumn{2}{|l|}{ Cigarettes per day } \\
\hline 5 or less & $848(29.3 \%)$ \\
\hline $6-10$ & $815(28.2 \%)$ \\
\hline $11-15$ & $387(13.4 \%)$ \\
\hline $16-20$ & $585(20.2 \%)$ \\
\hline $21-30$ & $157(5.4 \%)$ \\
\hline$>30$ & $102(3.5 \%)$ \\
\hline \multicolumn{2}{|l|}{ First cigarette of the day } \\
\hline$<5 \mathrm{~min}$ & $735(25.4 \%)$ \\
\hline $6-30 \mathrm{~min}$ & $918(31.7 \%)$ \\
\hline $31-60 \mathrm{~min}$ & $453(15.6 \%)$ \\
\hline$>60 \mathrm{~min}$ & $776(26.8 \%)$ \\
\hline Heaviness of Smoking Index (HSI-cig) & $2.11(1.6 \%)$ \\
\hline
\end{tabular}

nicotine use frequency, with nicotine uses per day increasing by $53 \%$. Similarly, $65 \%$ reported increased nicotine dependence.

Table 4 shows the hierarchical models predicting combustible cigarette dependence (HSI-cig) and total nicotine dependence (HSI-nic) after onset of vaping. Post-vaping combustible cigarette dependence (HSI-cig) was positively associated with lower education, more years smoking, higher HSI pre-vaping, fewer days vaping per month, and using e-liquids with higher nicotine concentration. The final model showed a good fit $(\mathrm{F}[13,2570]=76.056, p<.001)$ and explained $27.8 \%$ of the total variance. Regarding HSI-nic, younger age, higher HSI pre-vaping, more days vaping per month, taking more puffs per vaping session, using e-liquids with higher nicotine concentration, and more months using e-cigarettes were significantly associated with higher total nicotine dependence. The final model showed a good fit $(\mathrm{F}[13,2584]=61.701$, $p<.001)$ and explained $23.7 \%$ of the total variance.

\section{Discussion}

In this study, we examined, in a large sample of dual users of combustible and e-cigarettes, self-reported changes in smoking rates and
Table 2. Electronic Cigarette (E-Cigarette) Use Characteristics

\begin{tabular}{lr}
\hline & $n(\%)$ \\
\hline Time since started using e-cigarettes & \\
6 months or less & $450(15.5)$ \\
$7-12$ months ago & $417(14.4)$ \\
13-24 months ago & $749(25.9)$ \\
More than 24 mo ago & $1279(44.2)$ \\
Most important reason started e-cigarettes & \\
To use when can't smoke & $273(9.5)$ \\
Help quit smoking tobacco & $1309(45.6)$ \\
Help cut down amount of tobacco & $645(22.5)$ \\
Health concerns smoking tobacco & $348(12.1)$ \\
Curiosity & $143(5.0)$ \\
Recommendations & $78(2.7)$ \\
Other & $74(2.6)$ \\
Frequency of e-cigarette use & \\
No use in the past month & $16(0.6)$ \\
1-5 times a month & $125(4.3)$ \\
1-3 days a week & $304(10.5)$ \\
4-6 days a week & $448(15.5)$ \\
7 days a week & $2003(69.2)$
\end{tabular}

Times per day using e-cigarettes

$1-4$ times

$299(10.3)$

$5-9$ times

$327(11.3)$

$10-14$ times

$253(8.7)$

$15-19$ times

$211(7.3)$

20-29 times

$138(4.8)$

$>30$ times

$245(8.5)$

Continuously throughout the day

$1418(49.0)$

No use in past month

$4(0.1)$

First e-cigarette of the day

$\leq 5 \mathrm{~min}$

$842(29.4)$

6-30 min

$31-60 \mathrm{~min}$

$1003(35.0)$

$421(14.7)$

$>60 \mathrm{~min}$

$599(20.9)$

Number of puffs per e-cigarette

1-10

$569(57.5)$

11-20

$203(20.5)$

$77(7.8)$

$21-30$
+30

$141(14.2)$

Strength of nicotine concentration

$0 \mathrm{mg}$

$44(1.5)$

$1-3 \mathrm{mg}$

$1218(42.2)$

4-8 mg

9-15 mg

16-24 mg

$888(30.8)$

$296(10.3)$

$288(10.0)$

$+24 \mathrm{mg}$

$48(1.7)$

Don't know

$104(3.6)$

Type of e-cigarette used most often

Disposable

69 (2.4)

Cartridge

$92(3.2)$

Refillable

$2166(74.8)$

Other

$343(11.8)$

dependence, as well as total nicotine use and dependence, before and after initiating vaping. Findings showed that dual users significantly decreased their smoking and their combustible cigarette-based nicotine dependence. However, total nicotine uses per day and total nicotine dependence increased significantly from pre- to post-vaping.

Similar to previous studies with dual users, ${ }^{17,21}$ participants reported a significant decrease in number of cigarettes per day since onset of vaping. Thus, to the extent that e-cigarette use is less toxic than smoking, ${ }^{37}$ dual use might be expected to confer some decreased health risk compared to combustible cigarette smoking alone. 
Switching from tobacco cigarettes to e-cigarettes, even partially, is associated with a significant reduction in exposure to the carcinogens and toxicants in tobacco cigarette smoke. For example, studies have found that dual users who reduce their cigarette smoking experience a proportionate reduction in carbon monoxide and urine biomarkers of exposure to potentially harmful constituents..$^{38,39}$ However, in the long-term, smoking reduction, via dual use or otherwise, appears to provide little overall health benefit, ${ }^{40,41}$ and even low-frequency smokers have significantly elevated mortality compared to never smokers. ${ }^{42}$ Thus, any significant harm reduction associated with e-cigarette use is likely realized only after the complete cessation of combustible tobacco smoking.

Results of this study suggest that combustible cigarette dependence decreased after the onset of vaping, but total nicotine intake and dependence appear to have increased from pre- to post-vaping. This increase in nicotine dependence as a whole could indicate that it may be more difficult for dual users to quit all nicotine use compared to when they were only smoking cigarettes. However, some recent studies showed that dual users report that they have quit smoking over time. ${ }^{17,18,22}$ Etter and Bullen ${ }^{17}$ found that $46 \%$ of vapers who were daily or occasional smokers at baseline reported that they quit smoking after 12 months. In a later study with regular vapers, among the subsample of daily smokers, $28 \%$ reported that they quit

Table 3. Comparison of Total Score for Nicotine Frequency and Nicotine Dependence Pre- and Post- Vaping

\begin{tabular}{lrrrrrr}
\hline & \multicolumn{1}{c}{ Pre-vaping } & & Post-vaping & & \\
\cline { 2 - 3 } & $M(S D)$ & & $M(S D)$ & & $p$ & $d$ \\
\hline CPD & $19.2(9.0)$ & & $11.2(8.0)$ & $<.0001$ & .859 \\
HSI-cig & $3.6(1.5)$ & & $2.1(1.6)$ & $<.0001$ & .862 \\
Nicotine frequency & $19.3(9.0)$ & & $29.5(8.6)$ & $<.0001$ & -.945 \\
HSI-nic & $3.6(1.5)$ & & $4.7(1.4)$ & $<.0001$ & -.729 \\
\hline
\end{tabular}

M: mean; SD: Standard Deviation; CPD: Cigarettes per day; HSI-cig: Heaviness of Smoking Index for cigarettes; HSI-nic: Heaviness of Smoking Index for combined combustible and electronic cigarettes. smoking combustible cigarettes after 12 months. ${ }^{18}$ Zhuang et al. ${ }^{22}$ found that dual users who continued vaping were more likely to report having quit smoking over time compared to those who stopped using e-cigarettes or never used them.

Thus, although there may be an overall increase in nicotine dependence upon the addition of vaping, for a subset of smokers dual use appears to be a transition period before quitting smoking. ${ }^{21,43}$ Although NRT use before quitting smoking can be beneficial in a similar way, ${ }^{44,45}$ some e-cigarettes are able to deliver nicotine with a greater speed similar to the nicotine delivered by a combustible cigarette. ${ }^{46,47}$ This may contribute to greater nicotine dependence compared to NRT.

Results from the hierarchical regression analyses highlight the importance of pre-vaping nicotine dependence and vaping frequency as the strongest predictors of combustible tobacco nicotine dependence and total nicotine dependence. Indeed, it has been observed that dual users, compared to exclusive vapers, are more likely to have a longer history of smoking ${ }^{30}$ and higher cotinine levels before the onset of vaping. ${ }^{48}$ It appears that smokers with high prevaping nicotine dependence are able to meet less of their nicotine needs via e-cigarettes. However, those who vape more often, despite increasing their overall nicotine dependence, seem to be more likely to reduce their combustible cigarette smoking. ${ }^{9,20,29,30}$

This study has several limitations. First, because individuals who had already successfully switched to exclusive e-cigarette use were not included in the study, we were unable to compare dual users versus successful quitters in their e-cigarette dependence and frequency of use. Second, we used self-reported retrospective pre-vaping data that were collected cross-sectionally. Although a pre-vaping assessment might have been more accurate, self-reported smoking is generally reliable. ${ }^{49,50}$ Third, the last categories of the variable number of cigarettes/e-cigarettes per day (eg, >30 cigarettes/times per day) are broader than the ranges for the rest of the categories (eg, 1-10 times per day). However, this is a problem of many standard tobacco use/dependence measures such as the Fagerström Test for Nicotine Dependence. Fourth, we used the HSI-nic to assess the combination of tobacco and e-cigarette nicotine intake. In the absence of a

Table 4. Summary of Hierarchical Multiple Regression Analysis for Variables Predicting HSI-cig and HSI-nic

\begin{tabular}{|c|c|c|c|c|c|c|}
\hline & \multicolumn{3}{|c|}{ HSI-cig } & \multicolumn{3}{|c|}{ HSI-nic } \\
\hline & Model 1 & Model 2 & Model 3 & Model 1 & Model 2 & Model 3 \\
\hline Variable & $\beta$ & $\beta$ & $\beta$ & $\beta$ & $\beta$ & $\beta$ \\
\hline Sex & -.01 & .02 & -.01 & $-.08 *$ & -.05 & -.03 \\
\hline Age & $.20 *$ & .03 & .02 & .04 & $-.14 *$ & $-.15 *$ \\
\hline Race & -.02 & .02 & .03 & -.04 & .01 & .01 \\
\hline Ethnicity & $.06^{*}$ & .02 & .02 & $.07 *$ & .03 & .03 \\
\hline Marital status & .00 & -.04 & -.02 & .02 & -.03 & -.02 \\
\hline Education & $-.11 *$ & $-.09 *$ & $-.09 *$ & $-.06^{*}$ & -.04 & -.04 \\
\hline Income & $-.08 *$ & $-.07 *$ & -.04 & -.02 & -.01 & -.02 \\
\hline Years smoking & & $.08 *$ & $.07 *$ & & .05 & .05 \\
\hline HSI pre-vaping & & $.40 *$ & $.41 *$ & & $.43 *$ & $.41 *$ \\
\hline Number days vaping & & & $-.26^{*}$ & & & $.16^{*}$ \\
\hline Puffs per e-cigarette session & & & .04 & & & $.08 *$ \\
\hline Nicotine strength & & & $.11^{*}$ & & & $.09^{*}$ \\
\hline Months using e-cigarettes & & & -.04 & & & $.11 *$ \\
\hline$R^{2}$ & .050 & .193 & .274 & .016 & .182 & .233 \\
\hline$F$ for change in $R^{2}$ & $20.384 *$ & $230.135 *$ & $72.615 *$ & $7.026^{*}$ & $262.669 *$ & $44.465 *$ \\
\hline
\end{tabular}

HSI: Heaviness of Smoking Index.

$* p<.008$ 
validated nicotine dependence measure for dual users at the time of the study, the HSI was modified under the assumption that vaping episodes were roughly equivalent to smoking episodes, as has been done previously. ${ }^{20,33}$ This measure was created ad hoc for the purposes of this study and its validity and reliability are unknown. The HSI was already a blunt measure of nicotine dependence, given that it treats all smoking episodes as equal, without taking into account factors such as nicotine delivery and smoking topography, which differ across smokers and cigarettes. Adding e-cigarettes with all their variety and differences in nicotine dose and device power ${ }^{46,51}$ most likely contributes additional variability that could reduce the validity of the measure. Nevertheless, the HSI has demonstrated impressive construct validity, including its prediction of smoking cessation. ${ }^{52,53}$ Nevertheless, more research is needed to characterize the nature of e-cigarette dependence, its relationship to cigarette dependence, and total nicotine dependence among dual users. Future longitudinal analyses of the ongoing study should add clarity.

In summary, our findings provide support for dual use as a means to reduce combustible cigarette consumption. However, they also suggest that total nicotine use and dependence increases among dual users. The impact of increased nicotine dependence on eventual cessation of either smoking or vaping is as yet unclear.

\section{Funding}

This work was supported by the National Institute on Drug Abuse of the National Institutes of Health [R01DA037961]. The content is solely the responsibility of the authors and does not necessarily represent the official views of the National Institutes of Health. This work has also been supported in part by the Biostatistics and Survey Methods Core Facilities at the H. Lee Moffitt Cancer Center and Research Institute, a National Cancer Institute-designated Comprehensive Cancer Center (P30CA76292).

\section{Declaration of Interests}

THB has received research funding and medications from Pfizer, Inc., in previous studies. TE is a paid consultant in litigation against the tobacco industry and is a named inventor on a patent regarding measurement of electronic cigarette user behavior. DD is a paid witness in litigation against tobacco companies.

\section{References}

1. Lee C, Yong HH, Borland R, McNeill A, Hitchman SC. Acceptance and patterns of personal vaporizer use in Australia and the United Kingdom: results from the International Tobacco Control survey. Drug Alcohol Depend. 2018;185:142-148.

2. Abrams DB, Glasser AM, Pearson JL, Villanti AC, Collins LK, Niaura RS. Harm minimization and tobacco control: reframing societal views of nicotine use to rapidly save lives. Annu Rev Public Health. 2018;39:193-213.

3. Harrell PT, Marquinez NS, Correa JB, et al. Expectancies for cigarettes, e-cigarettes, and nicotine replacement therapies among e-cigarette users (aka vapers). Nicotine Tob Res. 2015;17(2):193-200.

4. Simmons VN, Quinn GP, Harrell PT, et al. E-cigarette use in adults: a qualitative study of users' perceptions and future use intentions. Addict Res Theory. 2016;24(4):313-321.

5. Kalkhoran S, Glantz SA. E-cigarettes and smoking cessation in real-world and clinical settings: a systematic review and meta-analysis. Lancet Respir Med. 2016;4(2):116-128. doi: 10.1016/S2213-2600(15)00521-4

6. Glantz SA, Bareham DW. E-Cigarettes: use, effects on smoking, risks, and policy implications. Annu Rev Public Health. 2018;39:215-235.

7. Etter JF, Bullen C. Electronic cigarette: users profile, utilization, satisfaction and perceived efficacy. Addiction. 2011;106(11):2017-2028.
8. Zhu SH, Zhuang YL, Wong S, Cummins SE, Tedeschi GJ. E-cigarette use and associated changes in population smoking cessation: evidence from US current population surveys. BMJ. 2017;358:j3262.

9. Giovenco DP, Delnevo CD. Prevalence of population smoking cessation by electronic cigarette use status in a national sample of recent smokers. Addict Behav. 2018;76:129-134.

10. Levy DT, Yuan Z, Luo Y, Abrams DB. The relationship of e-cigarette use to cigarette quit attempts and cessation: insights from a large, nationally representative US survey. Nicotine Tob Res. 2017;20(8):931-939.

11. Caponnetto P, Campagna D, Cibella F, et al. EffiCiency and Safety of an eLectronic cigAreTte (ECLAT) as tobacco cigarettes substitute: a prospective 12-month randomized control design study. PLoS One. 2013;8(6):e66317.

12. Bullen C, Howe $C$, Laugesen $M$, et al. Electronic cigarettes for smoking cessation: a randomised controlled trial. Lancet. 2013;382(9905):1629-1637.

13. Hartmann-Boyce J, McRobbie H, Bullen C, Begh R, Stead LF, Hajek P. Electronic cigarettes for smoking cessation. Cochrane Database Syst Rev. 2016;9:CD010216.

14. Quickstats: Cigarette smoking status among current adult e-cigarette users, by age group —national health interview survey, United States, 2015. Mmwr Morb Mortal Wkly Rep. 2016;65:1177. Doi:Http://dx.Doi. Org/10.15585/mmwr.Mm6542a7

15. Weaver SR, Majeed BA, Pechacek TF, et al. Use of electronic nicotine delivery systems and other tobacco products among USA adults, 2014: results from a national survey. Int J Public Health. 2016;61(2):177-188.

16. Patel D, Davis KC, Cox S, et al. Reasons for current e-cigarette use among U.S. adults. Prev Med. 2016;93:14-20.

17. Etter JF, Bullen C. A longitudinal study of electronic cigarette users. Addict Behav. 2014;39(2):491-494.

18. Etter JF. Electronic cigarette: a longitudinal study of regular vapers. Nicotine Tob Res. 2017;20(8):912-922. doi: 10.1093/ntr/ntx132

19. Nayak P, Pechacek TF, Weaver SR, Eriksen MP. Electronic nicotine delivery system dual use and intention to quit smoking: Will the socioeconomic gap in smoking get greater? Addict Behav. 2016;61:112-116.

20. Rass O, Pacek LR, Johnson PS, Johnson MW. Characterizing use patterns and perceptions of relative harm in dual users of electronic and tobacco cigarettes. Exp Clin Psychopharmacol. 2015;23(6):494-503.

21. Manzoli L, Flacco ME, Ferrante M, et al.; ISLESE Working Group. Cohort study of electronic cigarette use: effectiveness and safety at 24 months. Tob Control. 2017;26(3):284-292.

22. Zhuang YL, Cummins SE, Sun JY, Zhu SH. Long-term e-cigarette use and smoking cessation: a longitudinal study with US population. Tob Control. 2016;25(Suppl 1):i90-i95.

23. Young-Wolff KC, Klebaner D, Folck B, et al. Documentation of e-cigarette use and associations with smoking from 2012 to 2015 in an integrated healthcare delivery system. Prev Med. 2018;109:113-118.

24. Weaver SR, Huang J, Pechacek TF, et al. Are electronic nicotine delivery systems helping cigarette smokers quit? Evidence from a prospective cohort study of U.S. adult smokers, 2015-2016. PLoS One. 2018;13(7):e0198047.

25. Rostron BL, Schroeder MJ, Ambrose BK. Dependence symptoms and cessation intentions among US adult daily cigarette, cigar, and e-cigarette users, 2012-2013. BMC Public Health. 2016;16(1):814.

26. Orellana-Barrios MA, Payne D, Mulkey Z, Nugent K. Electronic cigarettes-a narrative review for clinicians. Am J Med. 2015;128(7):674-681.

27. Rüther T, Wissen F, Linhardt A, Aichert DS, Pogarell O, de Vries H. Electronic cigarettes-attitudes and use in Germany. Nicotine Tob Res. 2016;18(5):660-669.

28. Nardone N, Ko J, St. Helen G, Benowitz NL. Nicotine intake, dependence, and characteristics of electronic cigarette and dual users. Tob Regul Sci. 2019;5(1):27-35.

29. Morean M, Krishnan-Sarin S, O'Malley SS. Comparing cigarette and e-cigarette dependence and predicting frequency of smoking and e-cigarette use in dual-users of cigarettes and e-cigarettes. Addict Behav. 2018;87:92-96. 
30. Farsalinos KE, Romagna G, Voudris V. Factors associated with dual use of tobacco and electronic cigarettes: a case control study. Int J Drug Policy. 2015;26(6):595-600.

31. Etter JF, Eissenberg T. Dependence levels in users of electronic cigarettes, nicotine gums and tobacco cigarettes. Drug Alcohol Depend. 2015;147:68-75.

32. Browne M, Todd DG. Then and now: consumption and dependence in e-cigarette users who formerly smoked cigarettes. Addict Behav. 2018;76:113-121.

33. Foulds J, Veldheer S, Yingst J, et al. Development of a questionnaire for assessing dependence on electronic cigarettes among a large sample of ex-smoking e-cigarette users. Nicotine Tob Res. 2015;17(2):186-192.

34. Meltzer LR, Simmons VN, Sutton SK, et al. A randomized controlled trial of a smoking cessation self-help intervention for dual users of tobacco cigarettes and e-cigarettes: intervention development and research design. Contemp Clin Trials. 2017;60:56-62.

35. Heatherton TF, Kozlowski LT, Frecker RC, Rickert W, Robinson J. Measuring the heaviness of smoking: using self-reported time to the first cigarette of the day and number of cigarettes smoked per day. $\mathrm{Br} J$ Addict. 1989;84(7):791-799.

36. Heatherton TF, Kozlowski LT, Frecker RC, Fagerström KO. The Fagerstrom Test for Nicotine Dependence: a revision of the Fagerström Tolerance Questionnaire. Br J Addict. 1991;86(9):1119-1127.

37. National Academies of Sciences, Engineering, and Medicine. Public Health Consequences of e-cigarettes. Washington, DC: National Academies Press; 2018.

38. O'Connell G, Graff DW, D'Ruiz CD. Reductions in biomarkers of exposure (BoE) to harmful or potentially harmful constituents (HPHCs) following partial or complete substitution of cigarettes with electronic cigarettes in adult smokers. Toxicol Mech Methods. 2016;26(6):443-454.

39. D'Ruiz CD, Graff DW, Robinson E. Reductions in biomarkers of exposure, impacts on smoking urge and assessment of product use and tolerability in adult smokers following partial or complete substitution of cigarettes with electronic cigarettes. BMC Public Health. 2016;16(1):543.

40. Shahab L, Goniewicz ML, Blount BC, et al. Nicotine, carcinogen, and toxin exposure in long-term e-cigarette and nicotine replacement therapy users: a cross-sectional study. Ann Intern Med. 2017;166(6):390-400.

41. Pisinger C, Godtfredsen NS. Is there a health benefit of reduced tobacco consumption? A systematic review. Nicotine Tob Res. 2007;9(6):631-646.

42. Inoue-Choi M, Liao LM, Reyes-Guzman C, et al. Association of long-term, low-intensity smoking with all-cause and cause-specific mortality in the National Institutes of Health-AARP diet and health study. JAMA Intern Med. 2017;177(1):87-95.
43. Sussan TE, Shahzad FG, Tabassum E, et al. Electronic cigarette use behaviors and motivations among smokers and non-smokers. BMC Public Health. 2017;17(1):686.

44. Fagerström KO, Tejding R, Westin A, Lunell E. Aiding reduction of smoking with nicotine replacement medications: hope for the recalcitrant smoker? Tob Control. 1997;6(4):311-316.

45. Lindson N. Cochrane reviews-in their own words. Reduction versus abrupt cessation in smokers who want to quit. J Evid Based Med. 2010;3(2):133.

46. Wagener TL, Floyd EL, Stepanov I, et al. Have combustible cigarettes met their match? The nicotine delivery profiles and harmful constituent exposures of second-generation and third-generation electronic cigarette users. Tob Control. 2017;26(e1):e23-e28.

47. Hiler M, Breland A, Spindle T, et al. Electronic cigarette user plasma nicotine concentration, puff topography, heart rate, and subjective effects: influence of liquid nicotine concentration and user experience. Exp Clin Psychopharmacol. 2017;25(5):380-392.

48. McRobbie H, Phillips A, Goniewicz ML, et al. Effects of switching to electronic cigarettes with and without concurrent smoking on exposure to nicotine, carbon monoxide, and acrolein. Cancer Prev Res (Phila). 2015;8(9):873-878.

49. Caraballo RS, Giovino GA, Pechacek TF, Mowery PD. Factors associated with discrepancies between self-reports on cigarette smoking and measured serum cotinine levels among persons aged 17 years or older: third National Health and Nutrition Examination Survey, 1988-1994. Am J Epidemiol. 2001;153(8):807-814.

50. Hudmon KS, Pomerleau CS, Brigham J, Javitz H, Swan GE. Validity of retrospective assessments of nicotine dependence: a preliminary report. Addict Behav. 2005;30(3):613-617.

51. Rudy AK, Leventhal AM, Goldenson NI, Eissenberg T. Assessing electronic cigarette effects and regulatory impact: challenges with user selfreported device power. Drug Alcohol Depend. 2017;179:337-340.

52. Kozlowski LT, Porter CQ, Orleans CT, Pope MA, Heatherton T. Predicting smoking cessation with self-reported measures of nicotine dependence: FTQ, FTND, and HSI. Drug Alcohol Depend. 1994;34(3):211-216.

53. Borland R, Yong HH, O'Connor RJ, Hyland A, Thompson ME. The reliability and predictive validity of the Heaviness of Smoking Index and its two components: findings from the International Tobacco Control Four Country study. Nicotine Tob Res. 2010;12 (Suppl): S45-S50. 